\title{
$X$. Manejo nutricional en los programas de rehabilitación respiratoria de los pacientes con enfermedad pulmonar obstructiva crónica
}

\author{
XIMENA ORELLANA G.* y LAURA MENDOZA I.**
}

\author{
Nutritional management in pulmonary rehabilitation programs for patients with \\ chronic obstructive pulmonary disease
}

It is estimated that at least one third of patients with moderate to severe Chronic Obstructive Pulmonary Disease (COPD) have a significant alteration of their body composition, which is associated with deleterious clinical effects and higher mortality. However, there is a lack of evidence to support that dietary, pharmacological or both nutritional interventions have significant clinical benefits in COPD patients who are participating in a pulmonary rehabilitation program. At the present time the recommendation is to establish a nutritional diagnosis of the COPD patients from the beginning of the pulmonary rehabilitation program using the measurement of body mass index (BMI) and the estimation of the nutritional risk, which means a significant weight loss during a given period of time, followed by an individualized dietary and educational supplementation guide during at least 12 weeks.

Key words: Body composition, nutritional supplementation, respiratory rehabilitation, body mass index, nutritional risk, fat free mass.

\section{Resumen}

Se estima que al menos un tercio de los pacientes con enfermedad pulmonar obstructiva crónica moderada y severa tienen una alteración significativa en su composición corporal, lo cual se asocia con efectos clínicos deletéreos y con una mayor mortalidad. Sin embargo, hay evidencia insuficiente para respaldar que los pacientes que participan en un programa de rehabilitación respiratoria se beneficien en forma significativa de las intervenciones nutricionales dietarias, farmacológicas o la asociación de ambas, aunque aparentemente los últimos estudios publicados parecen sugerirlo. La recomendación actual es establecer desde que el paciente ingresa a un programa de rehabilitación respiratoria tanto el diagnóstico nutricional mediante la determinación del índice de masa corporal como el riesgo nutricional que corresponde a una baja involuntaria significativa de peso en determinado intervalo de tiempo, entregando al paciente un plan de alimentación y educación individualizado que debiera tener una duración mínima de 12 semanas.

Palabras clave: Composición corporal, suplementación nutricional, rehabilitación respiratoria, índice de masa corporal, riesgo nutricional, masa magra.

\section{Introducción}

Una proporción significativa de los pacientes con enfermedades respiratorias crónicas tiene alteración en su composición corporal siendo la Enfermedad Pulmonar Obstructiva Crónica (EPOC) la entidad respiratoria crónica mejor estudiada en este aspecto. Se estima que un tercio de los pa- cientes con EPOC moderado a severo tienen una relación peso/talla bajo lo normal ${ }^{1}$. Dicho porcentaje se incrementa aun más (de $32 \%$ a $63 \%$ ) en el grupo de pacientes que requieren ingresar a un programa de rehabilitación pulmonar².

Se reconoce actualmente que la composición corporal es un marcador sistémico de gravedad en los pacientes con $\mathrm{EPOC}^{3}$. De hecho, en el es-

* Servicio de Nutrición del Hospital Clínico de la Universidad de Chile.

** Jefe de Sección Neumología, Departamento de Medicina del Hospital Clínico de la Universidad de Chile. 
tudio de Schols y cols ${ }^{4}$, publicado en el año 2005 se demostró que la reducción de la masa libre de grasa es un predictor independiente de mortalidad en los pacientes con EPOC.

Considerando la relevancia de la composición corporal en la EPOC, las guías elaboradas por la American Thoracic Society (ATS) en conjunto con la European Respiratory Society (ERS) y las guías de la American Association of Cardiovascular and Pulmonary Rehabilitation (AACVPR) y American College of Chest Physicians (ACCP) recomiendan la incorporación del apoyo nutricional dentro de los programas de rehabilitación pulmonar $^{2,5}$.

La justificación para identificar y tratar las alteraciones de la composición corporal en los pacientes con enfermedad pulmonar crónica se basa en los siguientes puntos: 1) Su elevada prevalencia y asociación con morbilidad y mortalidad; 2) Los altos requerimientos energéticos durante el entrenamiento muscular en los programas de rehabilitación pulmonar que pueden agravar aún más estas anomalías (sin suplementos); y 3) Los mayores beneficios potenciales que se obtendrían de un programa de entrenamiento estructurado asociado al apoyo nutricional ${ }^{2}$.

Con respecto al método más apropiado para determinar el diagnóstico nutricional de los pacientes que ingresan a un programa de rehabilitación respiratoria se recomienda emplear simplemente la estimación obtenida con el Índice de masa corporal (IMC) $)^{2}$ que corresponde al peso en kilogramos dividido por la estatura en metros, elevada al cuadrado $\left(\mathrm{kg} / \mathrm{m}^{2}\right)$. De esta forma se pueden establecer diferentes categorías de IMC para sujetos adultos y adultos mayores de ambos sexos (Tabla 1). Para los adultos mayores $(\geq 65$ años) se consideran rangos diferentes y valores más altos de IMC dado que ante un evento agudo pueden perder más peso con mayor facilidad que el resto de los adultos y también les es más difícil lograr recuperarlo ${ }^{6}$.

Tabla 1. Diagnóstico nutricional de los pacientes con enfermedad pulmonar obstructiva crónica según índice de masa corporal y grupo etario

\begin{tabular}{|lcc|}
\hline Diagnóstico & Adultos* & Adultos mayores* \\
Bajo peso & $<21$ & $<23$ \\
Normal & $21-25$ & $23-28$ \\
Sobrepeso & $>25 \mathrm{y}<30$ & $>28 \mathrm{y}<30$ \\
Obesidad & $>30$ & $>30$ \\
\hline
\end{tabular}

*Valores de IMC expresados en $\mathrm{kg} / \mathrm{m}^{2}$.
Tanto en la evaluación inicial como en el seguimiento de los pacientes en su aspecto nutricional se considera también relevante determinar el riesgo nutricional que corresponde a una baja de peso involuntaria mayor al $10 \%$ de su peso inicial en un período de seis meses o mayor a $5 \%$ en un mes $^{2}$.

Sin embargo, la estimación del peso corporal y el IMC no reflejan fehacientemente la composición corporal de los pacientes crónicos ${ }^{3}$. El parámetro más importante es la masa libre de grasa (MLG) o masa magra que corresponde a la masa celular corporal (órganos, músculos, huesos) y el agua. En condiciones de estabilidad clínica, la medición de la MLG puede ser utilizada para estimar la masa celular corporal. Para determinar en forma precisa si los pacientes tienen realmente disminución de la MLG existen distintas alternativas, siendo la más fácil y económica la utilización de medidas antropométricas como la medición de los pliegues cutáneos, no obstante, esta forma de estimación es imprecisa por lo que actualmente no es recomendada. Otras modalidades para la determinación de la composición corporal corresponden a la bioimpedanciometría corporal y el DEXA sigla en inglés de Dual Energy $X$ Ray Absorptiometry. Ambas técnicas son muy confiables pero de costo muy elevado y se utilizan en muy pocos centros de rehabilitación, habitualmente dentro del campo de la investigación ${ }^{2,7,8}$.

\section{Preguntas}

1. ¿El apoyo nutricional es beneficioso para los pacientes con enfermedad pulmonar obstructiva crónica en rehabilitación respiratoria?

2. ¿Cuál es el mejor soporte nutricional para los pacientes con EPOC sometidos a un programa de rehabilitación respiratoria?

\section{Pacientes}

Pacientes con enfermedad pulmonar obstructiva crónica estable en un programa de rehabilitación respiratoria.

\section{Intervención}

Evaluación y suplementación nutricional oral en pacientes con enfermedad pulmonar obstructiva crónica.

\section{Objetivo}

Evaluar si la suplementación nutricional tiene impacto en la disnea, aumenta la capacidad de realizar ejercicio y mejora la calidad de vida en los pacientes con EPOC. 


\section{Resumen de la evidencia}

La biblioteca Cochrane llevó a cabo una revisión sistemática publicada en el año 2000 que luego fue actualizada en el año 2005. Esta revisión incluyó once estudios clínicos aleatorizados que incluyeron a un total de 352 pacientes, ocho de los estudios fueron considerados de alta calidad, pero sólo dos de estos ensayos clínicos eran doble ciego. Ellos concluyen que no se observaron beneficios en la antropometría, la función pulmonar y la capacidad de ejercicio con el soporte nutricional de al menos dos semanas de duración en pacientes con EPOC estable?

Sin embargo, en el año 2009, Weekes y cols ${ }^{10}$, publicaron un estudio multicéntrico inglés que consistió en un ensayo clínico aleatorio con grupo control no ciego en pacientes con EPOC estable. Los autores observaron efectos estadísticamente significativos en el grupo intervenido (apoyo nutricional) en cuanto a ganancia de peso y en la calidad de vida, sin evidenciar efectos significativos en la masa magra, lo que puede ser explicado porque en este estudio la intervención nutricional fue realizada sin acompañarse de rehabilitación respiratoria.

Creutzberg y cols ${ }^{11}$, estudiaron los efectos de la suplementación nutricional oral durante ocho semanas en el curso de la rehabilitación respiratoria en pacientes desnutridos con EPOC. Ellos encontraron un incremento en la masa magra, la fuerza muscular y el rendimiento con el ejercicio y en la calidad de vida, pero es importante considerar que este estudio careció de grupo control.

Baldi y $\operatorname{cols}^{12}$, en un estudio aleatorio investigaron el efecto del aporte de aminoácidos esenciales sobre el peso corporal y la MLG. En este ensayo clínico, 28 pacientes con EPOC y mala nutrición ingresaron a un programa de doce semanas de rehabilitación respiratoria. Los autores observaron un incremento significativo del peso corporal y de la MLG en el grupo experimental comparado con el grupo control.

Por último, recientemente fue publicado por Sugawara y $\operatorname{cols}^{13}$, un estudio clínico aleatorio en que investigaron los efectos de la suplementación nutricional en combinación con ejercicios de baja intensidad en pacientes con EPOC moderado a severo y desnutrición (IMC $<19 \mathrm{~kg} / \mathrm{m}^{2}$ ). Los autores encontraron que esta combinación se asoció significativamente a aumento de peso, masa muscular y consumo de energía, así también, mejoría en la capacidad de ejercicio y la calidad de vida. Por otra parte, las principales citoquinas inflamatorias sistémicas (TNF $\alpha$, IL-6 e IL-8) disminuyeron significativamente. Los resultados del estudio sugieren un rol potencial de la combinación de suplementos nutricionales y el ejercicio de baja intensidad en el tratamiento de los pacientes desnutridos portadores de EPOC.

El apoyo nutricional tiene como objetivo principal conseguir que los pacientes mantengan un peso corporal y masa magra muscular dentro de rangos aceptables. Existe una alta proporción de pacientes con EPOC que al momento de ingresar a los programas de rehabilitación pulmonar presentan depleción nutricional. Además, se tiene que tener en cuenta que el ejercicio físico al que serán sometidos estos pacientes, durante el programa de rehabilitación respiratoria, implicará un mayor gasto energético comparado con el que tenían previamente. No obstante, no está claramente establecido el tipo de soporte nutricional que produce beneficios clínicos en los pacientes con EPOC y malnutrición. Las intervenciones nutricionales que se pueden realizar las podemos clasificar en: 1) Suplementación calórica y/o proteica $\left.{ }^{10} ; 2\right)$ Intervenciones fisiológicas o farmacológicas; y 3) Combinaciones de estas².

En cuanto a la suplementación nutricional, el efecto beneficioso del aporte de aminoácidos esenciales sobre el peso corporal y la MLG ha sido demostrado por Baldi y cols ${ }^{12}$. En este estudio existe evidencia del potencial de los aminoácidos esenciales (AAE) complementarios circulantes en la regulación de la señales mediadas por la insulina en el metabolismo de las proteínas y la glucosa. Estos resultados nos proporcionan una justificación para el uso de AAE como un valioso complemento de los ejercicios físicos en los programas de rehabilitación respiratoria destinados a estabilizar o incluso revertir los efectos negativos de la pérdida de la masa magra corporal en los pacientes portadores de EPOC.

La creatina es un suplemento nutricional que en forma rápida se transforma en fosfocreatina en el músculo esquelético, constituyendo una fuente de alta energía para el músculo. Se ha demostrados en atletas y sujetos sanos que la suplementación con creatina tiene efectos beneficiosos en la capacidad de ejercicio y la masa magra. No obstante, su utilización en pacientes con EPOC no entrega beneficios extras durante la rehabilitación respiratoria. Esto ha quedado demostrado por Deacon y $\operatorname{cols}^{14}$, en un ensayo clínico controlado y doble ciego que incluyó a cien pacientes con EPOC sometidos a un programa de rehabilitación respiratoria. El grupo experimental recibió suplementación con creatina durante siete semanas. Los autores no observaron diferencias significativas en la fuerza muscular y en la capacidad de ejercicio al comparar ambos grupos. 
Con respecto a las intervenciones farmacológicas, existen ensayos clínicos publicados que investigan el efecto del uso de hormona del crecimiento, esteroides anabólicos como la nandrolona, el acetato de megestrol o de la testosterona. Sin embargo, éstos no han demostrado claros beneficios sobre la composición corporal y capacidad de ejercicio de los pacientes con EPOC ${ }^{2,7,8}$. Se necesitan más estudios para determinar si aportan beneficios clínicos, sus efectos a largo plazo y su relación costo-efectividad.

En los pacientes con sobrepeso u obesidad se recomienda indicar un plan de alimentación con educación alimentaria, restricción calórica, fomento de la pérdida de peso y apoyo psicológico $^{2}$. Si bien, aún no existe una meta establecida en relación a la magnitud de la pérdida de peso que se logra obtener después de la rehabilitación respiratoria, la rehabilitación integral de las personas obesas puede conducir a la pérdida de peso, mejoría del estado funcional y calidad de vida.

\section{Grado de recomendación}

1. El apoyo nutricional debería ser considerado como parte del programa de rehabilitación pulmonar (calidad de la evidencia $\mathrm{C}$, fuerza de la recomendación moderada).

2. No hay suficiente evidencia para realizar recomendaciones específicas en relación a las características que debe tener la suplementación nutricional aunque pareciera aportar ciertos beneficios a la rehabilitación pulmonar (sin recomendación al respecto).

3. En relación al uso de creatina en pacientes con EPOC, la evidencia es clara en que ésta carece de beneficios para los pacientes con EPOC (no se recomienda).

4. El uso de intervenciones de tipo farmacológicas no se recomiendan actualmente en los programas de rehabilitación pulmonar porque se carece de estudios que determinen su beneficio en el paciente con EPOC.

\section{Conclusión}

Existe evidencia clínica en relación a que el bajo peso corporal en pacientes con EPOC se asocia a deterioro de la función pulmonar, reducción de la masa muscular diafragmática, menor capacidad de ejercicio y mayor tasa de mortalidad en comparación con las personas adecuadamente alimentadas con esta enfermedad. Los estudios más recientes sugieren que el apoyo nutricional puede entregar beneficios para los pacientes que participan en un programa de rehabilitación respiratoria. Sin embargo, no existe suficiente información para recomendar el tipo, cantidad y la duración que debiera tener la intervención nutricional. Se recomienda establecer el diagnóstico nutricional por medio del índice de masa corporal (IMC) y el riesgo nutricional, junto con la elaboración de un plan de alimentación individual y educación alimentaria, considerando las comorbilidades, los factores socioeconómicos y culturales, con una duración mínima de 12 semanas o durante toda la permanencia en el programa de rehabilitación respiratoria.

\section{Bibliografía}

1.- ENGELEN M P, SCHOLS A M, BAKEN W C, WESSELING G J, WOUTERS E F. Nutritional depletion in relation to respiratory and peripheral skeletal muscle function in out-patients with COPD. Eur Respir J 1994; 7: 1793-7.

2.- NICI L, DONNER C, WOUTERS E, ZUWALLACK R, AMBROSINO N, BOURBEAU J, et al. American Thoracic Society/European Respiratory Society statement on pulmonary rehabilitation. Am J Respir Crit Care Med 2006; 173: 1390-413.

3.- DE BENEDETTO F, DEL PONTE A, MARINARI S, SPACONE A. In COPD patients, body weight excess can mask lean tissue depletion: a simple method of estimation. Monaldi Arch Chest Dis 2000; 55: 273-8.

4.- SCHOLS A M, BROEKHUIZEN R, WELINGSCHEEPERS C A, WOUTERS E F. Body composition and mortality in chronic obstructive pulmonary disease. Am J Clin Nutr 2005; 82: 53-9.

5.- RIES A L, BAULDOFF G S, CARLIN B W, CASABURI R, EMERY C F, MAHLER D A, et al. Pulmonary rehabilitation: Joint ACCP/AACVPR evidence-based clinical practice guidelines. Chest 2007; 131 (5 Suppl): 4S-42S.

6.- CASTILLO C, UAUY R, ATALAH E. Evaluación del estado nutricional en el anciano. Guías de alimentación para el adulto mayor. Bases para la acción. Ministerio de Salud 1999. p. 37-52.

7.- SHRIKRISHNA D, HOPKINSON N S. Skeletal muscle dysfunction in chronic obstructive pulmonary disease. Respiratory Medicine: COPD Update 2009; 5: 7-13.

8.- JACKSON A S, HOPKINSON N. Skeletal muscle in chronic obstructive pulmonary disease. Clinical Pulmonary Medicine 2009; 16: 61-67.

9.- FERREIRA I M, BROOKS D, LACASSE Y, GOLDSTEIN R S, WHITE J. Nutritional supplementation for stable chronic obstructive pulmonary disease. Cochrane Database Syst Rev 2005; (2): CD000998.

10.- WEEKES C E, EMERY P W, ELIA M. Dietary counse- 
lling and food fortification in stable COPD: a randomised trial. Thorax 2009; 64: 326-31.

11.- CREUTZBERG E C, WOUTERS E F, MOSTERT R, WELING-SCHEEPERS C A, SCHOLS A M. Efficacy

of nutritional supplementation therapy in depleted patients with chronic obstructive pulmonary disease. Nutrition 2003; 19: 120-7.

12.- BALDI S, AQUILANI R, PINNA G D, POGGI P, DE MARTINI A, BRUSCHI C. Fat-free mass change after nutritional rehabilitation in weight losing COPD: role of insulin, c-reactive protein and tissue hypoxia. Int J
Chron Obstruct Pulmon Dis 2010; 5: 29-39

13.- SUGAWARA K, TAKAHASHI H, KASAI C, KIYOKAWA N, WATANABE T, FUJII S, et al. Effects of nutritional supplementation combined with lowintensity exercise in malnourished patients with COPD. Respir Med 2010; 104: 1883-9.

14.- DEACON S J, VINCENT E E, GREENHAFF P L, FOX J, STEINER M C, SINGH S J, et al. Randomized controlled trial of dietary creatine as an adjunct therapy to physical training in chronic obstructive pulmonary disease. Am J Respir Crit Care Med 2008; 178: 233-9.

Correspondencia a:

Dra. Laura Mendoza I.

Sección Neumología, Departamento de Medicina,

Hospital Clínico Universidad de Chile.

E-mail:1mendoza@redclinicauchile.cl

Rev Chil Enf Respir 2011; 27: 139-143 